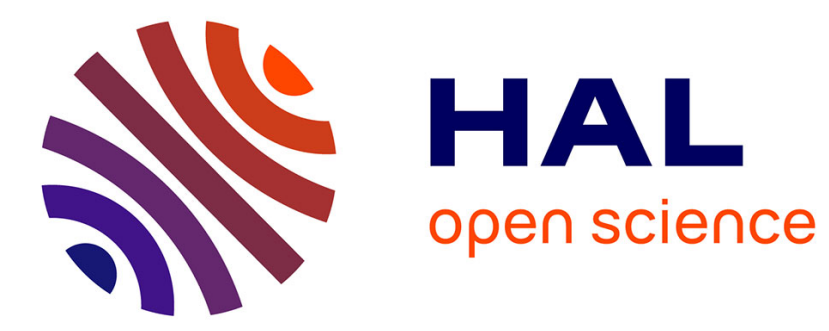

\title{
Conical optics: the solution to confine light
}

T. Grosjean, F.I. Baida, D. Courjon

\section{To cite this version:}

T. Grosjean, F.I. Baida, D. Courjon. Conical optics: the solution to confine light. Applied optics, 2007, 46 (11), pp.1994-2000. 10.1364/AO.46.001994 . hal-00216007

\section{HAL Id: hal-00216007 https://hal.science/hal-00216007}

Submitted on 17 Apr 2021

HAL is a multi-disciplinary open access archive for the deposit and dissemination of scientific research documents, whether they are published or not. The documents may come from teaching and research institutions in France or abroad, or from public or private research centers.
L'archive ouverte pluridisciplinaire HAL, est destinée au dépôt et à la diffusion de documents scientifiques de niveau recherche, publiés ou non, émanant des établissements d'enseignement et de recherche français ou étrangers, des laboratoires publics ou privés. 


\title{
Conical optics: the solution to confine light
}

\author{
T. Grosjean, F. Baida, and D. Courjon
}

\begin{abstract}
We compare the performances in terms of confinement and depth of field of spherical and conical optics. It turns out that, if the spherical optics is adapted to the usual parallel imaging, conical optics seems to be the optimized solution for systems based on scanning (sequential imaging). It is shown that the optimized confinement capability of conical optics is due to the ability of conical components to generate a single Bessel beam with high efficiency. The calculations are based on Weyl formulas.
\end{abstract}

\section{Introduction}

Beyond the improvement of the performances and potentialities of the inspection at submicrometer scale, scanning microscopy (scanning electron microscopy, ${ }^{1}$ confocal microscopy, ${ }^{2}$ etc.) has marked a turning point in imaging concepts. It has brought an alternative image forming procedure where the entire object field is not imaged at once anymore (the basic parallel imaging principle of conventional microscopes) but point by point by focusing waves onto the sample (a sequential imaging process).

With the development of lasers, the optical sequential process has found many applications in a wide array of technologies. In the past decades, a growing number of sequential processing systems have been developed in laser cutting, ${ }^{3}$ microfabrication and nanofabrication, ${ }^{4-6}$ nanolithography, ${ }^{7}$ data storage, ${ }^{8}$ etc.

By technological continuity, spherical optics (conventional lenses, objectives, etc.) has been adapted from the parallel to the sequential imaging concept. In this evolution, spherical optics has left its primary role of being a direct imaging device to be used as a focusing and collecting system. Therefore, spherical optics has been kept as the privileged means of fo-

The authors are with the Département d'Optique P. M. Duffieux, Institut FEMTO-ST, UMR CNRS 6174, Université de FrancheComté, 16 Route de Gray, 25030 Besançon cedex, France. T. Grosjean's e-mail address is thierry.grosjean@univ-fcomte.fr. cusing light in the expansion of sequential processes in a larger field of applications.

In this paper we demonstrate that sequential processing systems have to follow a different technological approach than those derived from the parallel imaging concept. If it is well understood that, in usual imaging, optical waves are mastered by spherical interfaces (the heritage of Huygens's theory), specific systems adapted to tightly focus light are required for scanning devices. We will see that the application of spherical optics in this task is disputable. We demonstrate here that conical optics is a valuable solution. The idea of the conical objective, which can be defined as a high-NA combination of cone lenses, also called axicons, ${ }^{9}$ will be introduced in this paper.

In Section 2 the vectorial focused field will be described analytically. The subsequent theoretical analysis of the focusing process will serve as a basis to introduce the solution we proposed to minimize focus lateral size (Section 3). Finally in Section 4 we will discuss why conical optics seems to be the right way to confine light.

\section{Focused Fields: Coherent Summation of Vectorial Bessel Beams}

Let us consider as a starting point of this study the particular focusing system depicted in Fig. 1. It is composed of a conventional objective microscope that is schematized by an abberation-free biconvex lens (BL) and of a solid immersion lens (SIL) set in front of the sample. The SIL 10 leads to a regime where the NA is larger than 1. The NA is limited by the lateral size of the circular exit pupil. Such a system is considered aplanetic; i.e., it fulfills the Abbe sine conditions. ${ }^{11}$

The expression of the field transmitted by such a device is generally written in the Debye approxima- 


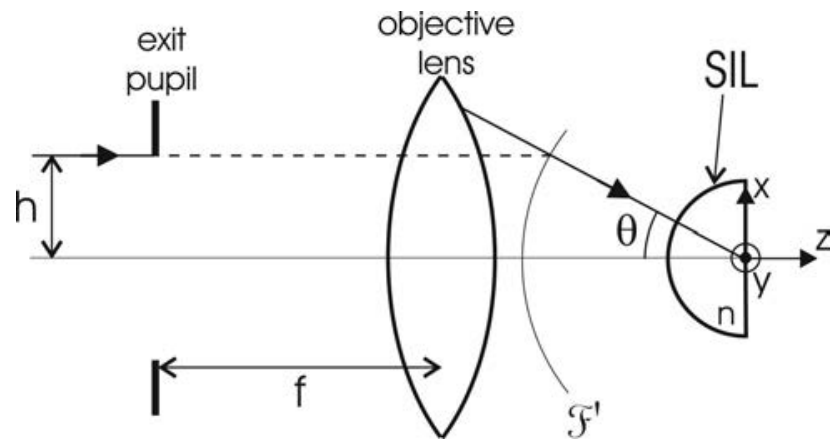

Fig. 1. Scheme of the considered focusing system consisting of an aplanetic focusing objective and a SIL.

tion (the edge diffraction at the exit pupil plane is neglected). In this case, focused fields, which are described by spherical wavefronts, are usually expanded in the plane wave spectrum by means of the Debye integral. ${ }^{12,13}$ Since the expression of the Debye integral is calculated asymptotically, the focus is imposed to be many wavelengths away from the output pupil. ${ }^{13}$ In this paper we choose to overcome this limitation by using the Weyl theorem ${ }^{14}$ to calculate the expression of the focused wave.

Owing to the Weyl theorem, the electric field of the spherical wave transmitted through the SIL can be rigorously expanded in the plane wave spectrum as follows:

$$
\mathbf{E}(\mathbf{r})=\frac{i}{2 \pi} \iint \frac{\mathbf{a}(u, v)}{w} \exp [i(u x+v y+w z)] \mathrm{d} u \mathrm{~d} v,
$$

where $u, v$, and $w$ are the wave vector components and $\mathbf{r}(x, y, z)$ defines the spatial position. We have $u^{2}+v^{2}+w^{2}=k^{2}$, where $k$ is the wavenumber.

Let us note that the Weyl and Debye integrals are similar. However, the expression of Eq. (1) is rigorously calculated with the Weyl theorem. Therefore the field expression remains valid in all the free space, whatever the focal length (or Fresnel number) of the focusing device.

The angular spectrum representation can be deduced from Eq. (1) as

$$
\begin{aligned}
\mathbf{E}(r, \xi)= & \frac{i k}{2 \pi} \int_{0}^{\pi / 2} \mathrm{~d} \theta \sin \theta \int_{0}^{2 \pi} \mathrm{d} \psi \mathbf{A}(\theta, \psi) \\
& \times \exp [i \alpha r \cos (\psi-\xi)+i w z],
\end{aligned}
$$

where $\theta, \psi$, and $\xi$ are azimuthal angles, and $\alpha^{2}=$ $u^{2}+v^{2}$.

Following the ray-tracing procedure proposed in Ref. 12, the expression of the parameter $\mathbf{A}(\theta, \psi)$ can be defined from the vectorial electric field distribution at the exit pupil plane. First it is assumed that the absorption and reflection within the BL are sufficiently weak to be neglected and, second, that the polarization state of the field that propagates through the BL remains unchanged. Function $\mathbf{A}(\theta, \psi)$ can be seen as the product of three terms:

$$
\mathbf{A}(\theta, \psi)=\sqrt{\cos \theta} f(\theta) \mathbf{P}(\theta, \psi) .
$$

Vector $\mathbf{P}(\theta, \psi)$ defines the polarization direction of each plane wave of angular frequency $(\theta, \psi)$. Coefficient $\sqrt{\cos \theta}$ is an obliquity coefficient obtained from the intensity law of geometrical optics. ${ }^{12}$ Function $f(\theta)$ is the transmittance function at the exit pupil plane. We have

$$
f(\theta)=L G[R(\theta)] \times \operatorname{circ}[R(\theta)-1],
$$

where $R$ is the radial spatial coordinate at the exit pupil plane. From the sine condition, we have $R=$ $\mathrm{n} \sin (\theta) / N A$.

Function $L G(R)$ provides a Laguerre-Gauss profile to the incident wave at the exit pupil plane. Function $\operatorname{circ}(R-1)$ models the circular pupil function, which limits the NA of the focusing system. In other words, this function defines the solid angle of the geometrical cone of light rays generated by the BL. From the Debye approximation, it is defined as

$$
\begin{aligned}
\operatorname{circ}(R-1) & =1 \text { if } R<1, \\
& =0 \text { if } R>1 .
\end{aligned}
$$

Let us note that, in the following, the diameter of the exit pupil will be chosen to fit the $1 / e$ width of the incident Laguerre-Gauss profiles. In this case, the effect of pupil diffraction on focusing is sufficiently weak to be negligible, validating the Debye approximation.

After calculations, the expression of the focused field transmitted through the SIL takes the following form:

$$
\mathbf{E}(r, \xi)=\frac{i k E_{0} T}{2 \pi} \int_{0}^{\pi / 2} \mathrm{~d} \theta \sin \theta \sqrt{\cos \theta} f(\theta) \mathbf{B B}(\theta, r, \xi),
$$

where $E_{0}$ and $T$ are constants. We have in linear, circular, and elliptical polarizations

$$
\begin{aligned}
B B_{x}= & \pi \exp [i w z]\left[\left(t_{s}+t_{p_{r}} \cos \theta\right) \cos \phi J_{0}(\alpha r)\right. \\
& +\left(t_{s}-t_{p_{r}} \cos \theta\right)(\cos \phi \cos 2 \xi \\
& \left.+a \sin \phi \sin 2 \xi) J_{2}(\alpha r)\right], \\
B B_{y}= & \pi \exp [i w z]\left[\left(t_{s}+t_{p_{r}} \cos \theta\right) a \sin \phi J_{0}(\alpha r)\right. \\
& -\left(t_{s}-t_{p_{r}} \cos \theta\right)(a \sin \phi \cos 2 \xi \\
& \left.-\cos \phi \sin 2 \xi) J_{2}(\alpha r)\right], \\
B B_{z}= & -2 i \pi \exp [i w z] \sin \theta t_{p_{z}}(\cos \phi \cos \xi \\
& +a \sin \phi \sin \xi) J_{1}(\alpha r),
\end{aligned}
$$


with $L G(R)=\exp \left[-R^{2}\right]$. Parameter $T$ defines the transmission through the exit semispherical interface of the SIL, whereas $t_{s}, t_{p_{r}}$, and $t_{p_{z}}$ characterize the transmission through its entrance flat interface, for the $s$ and $p$ components. Without any SIL, coefficients $T, t_{s}, t_{p_{p}}$, and $t_{p_{z}}$ are equal to 1 . Coefficients $a$ and $\phi$ define the polarization state at the exit pupil plane. In linear polarization, $a=1$ and angle $\phi$ specifies the polarization direction; in circular polarization, $a=i$ and $\phi=\pi / 4$, whereas in elliptic polarization $a=i$ and $\phi \neq \pi / 4$.

We have in radial polarization

$$
\begin{aligned}
& B B_{x}=-i \exp [i w z] t_{p_{r}} \cos \theta \cos \xi J_{1}(\alpha r), \\
& B B_{y}=-i \exp [i w z] t_{p_{r}} \cos \theta \sin \xi J_{1}(\alpha r), \\
& B B_{z}=\exp [i w z] t_{p_{z}} \sin \theta J_{0}(\alpha r),
\end{aligned}
$$

with $L G(R)=2(1.43 R) \exp \left[-(1.43 R)^{2}\right]$.

From Eqs. (8)-(10) and (11)-(13) and Refs. 15-17, it turns out that the expression of the vector field $\mathbf{B B}(\theta, r, \xi)$ is proportional to those describing the Bessel beams. Let us recall that Bessel beams can be defined as the coherent combination of plane waves whose wave vectors lie on a cone of half-angle $\theta .18$ Therefore they are the solutions of Eq. (2) over the azimuthal angle $\psi$. Finally, Eq. (7) points out that focused beams are the coherent superposition of Bessel beams whose aperture angles $\theta$ vary from 0 to $\theta_{m}=\arcsin (\mathrm{NA} / n)$, where $n$ is the optical index of the SIL. Angle $\theta_{m}$ is limited by the exit pupil lateral size. Let us note that a similar analysis has been given for space-time focusing of light pulses. ${ }^{19}$

\section{Bessel Beam: Result of Focusing Optimization}

In the context of system miniaturization, one of the current challenges for sequential processing systems is to reduce focal spots to the minimum achievable size. To this end, a large number of superresolution techniques has been developed to reduce the focus lateral width. In most cases, they consist of placing specific phase and/or amplitude filters at the exit pupil plane of the high-NA objectives. ${ }^{20-26}$ In this section, we propose a solution that yields to the highest confinement capability of focusing systems. Since the detailed procedure optimizes superresolution, it will be called focusing optimization.

According to diffraction laws, it is well known that the spot lateral extension is reduced by means of a high-pass angular frequency filtering. ${ }^{2,27}$ This procedure is carried out by attenuating or canceling the components of the lowest angular frequencies and keeping the highest ones in the angular spectrum. In our case, this can be easily simulated by replacing function $f(\theta)$ in Eq. (7) by $f^{\prime}(\theta, \psi)=f(\theta) F(\theta, \psi)$, where $F(\theta, \psi)$ is the filtering function. Owing to the cylindrical symmetry of the problem, filtering over angle $\psi$ has in principle no influence on spot size. We can write $F(\theta, \psi)=F(\theta)$. Therefore it turns out, from Section 2 , that the above-described filtering process can be alternatively seen as the selection in the focused field of the Bessel beams over the range of high values of angle $\theta$.

Following diffraction properties, focusing optimization implies that all the Bessel beams that constitute the focused beam are filtered out except the highestaperture-angle Bessel beam that carries the smallest light confinement. In this case, we have

$$
F(\theta)=\delta(R(\theta)-1) .
$$

Thus Bessel beams can be seen as the result of focusing optimization. In other words, the smallest confinement zones that can be generated optically through focusing are carried by Bessel beams.

Bessel beams are known as nondiffracting solutions of Maxwell's equations. ${ }^{28}$ Such a nondiverging property is attributable to the fact that the $z$ dependence of the field distributions (which generally defines diffractive spreading) is carried by the phase term $\exp [i w z]$. In the case of propagative Bessel beams ( $w$ is real), the field amplitude is $z$ nondependent, meaning that the beam shape remains unchanged along the propagation $z$ direction. In the evanescent case ( $w$ is imaginary), the $z$ dependence of the field amplitude is described by an evanescent decay that does not influence the beam transverse shape. Therefore focal depth becomes infinite in the propagative regime and is limited by the exponential decay of the field in the evanescent regime. Let us note that ideal theoretical Bessel beams have been treated here. In practice, such beams are not physically realizable since Bessel functions are not square integrable. In real cases, the focal depth is limited by the Bessel beam lateral width to ranges much higher than the Rayleigh distance of Gaussian beams. ${ }^{29-31}$ Therefore Bessel beams provide both the smallest light confinement (focusing optimization) and the longest focal depth that can be generated with focusing systems. Focusing optimization is systematically associated with the maximization of the depth of focus.

Figures 2 and 3 display the transverse $(x, y)$ and longitudinal $(x, z)$ cross sections of a radially polarized focused beam in the focusing optimization configuration and without angular frequency filtering. We note that radial polarization has interesting properties in the focusing procedure. ${ }^{17,32}$ In this example, two values for NA are considered: $\mathrm{NA}=\sin (\pi / 3)$ $=0.87$ (Fig. 2) and $\mathrm{NA}=1.5 \sin (\pi / 3)=1.73$ (Fig. 3). In the latter case, the behavior of the exponential decaying intensity distribution ( $I$ ) along the $z$ direction has been emphasized by displaying $I^{0.2}$ rather than $I$ [Figs. 3(c) and 3(d)]. One consequence is that the lateral fringes of the beams are more pronounced in these two figures.

In both regimes of NA, we observe that the spot size reduction is remarkable with Bessel beams. Moreover, the central interference fringe acts as a nondiverging stylus of light [see Figs. 2(d) and 3(d)]. In the evanescent regime [Fig. 3(d)], this fringe has been proposed to be used as a virtual (or immaterial) tip for near-field applications. ${ }^{17}$ It allows the exten- 


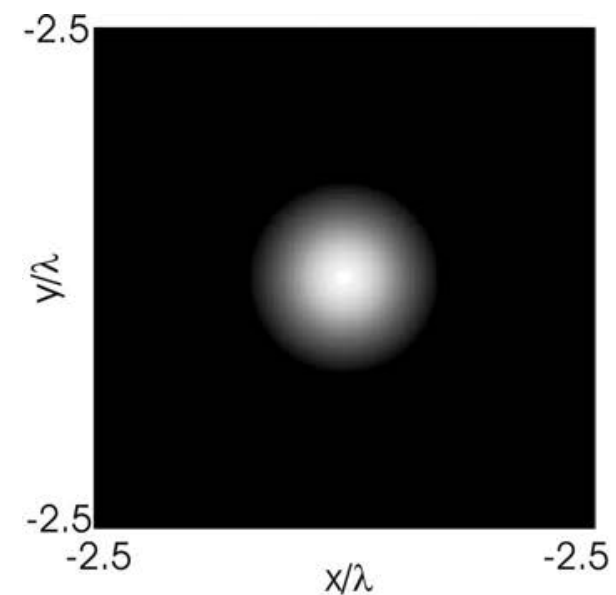

(a)

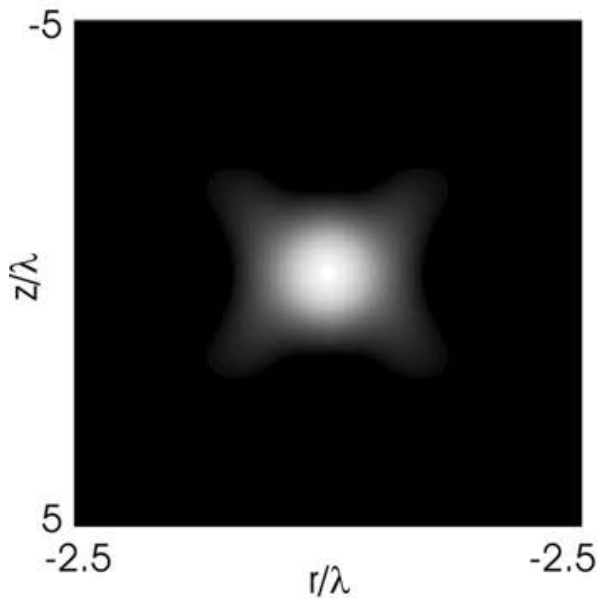

(c)

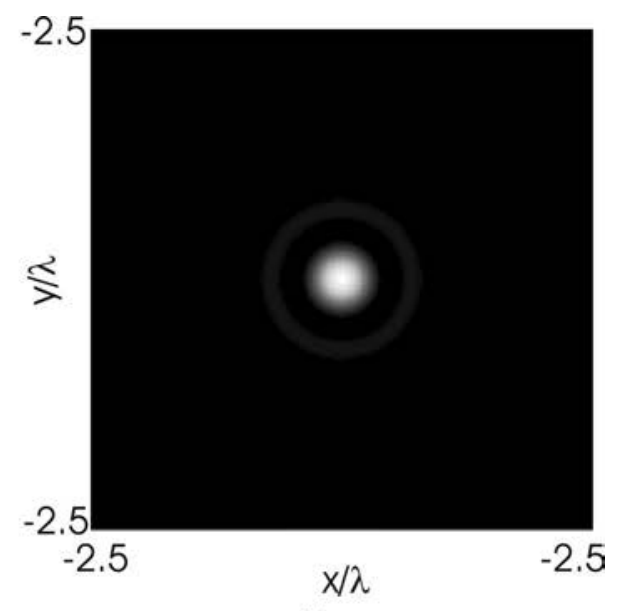

(b)

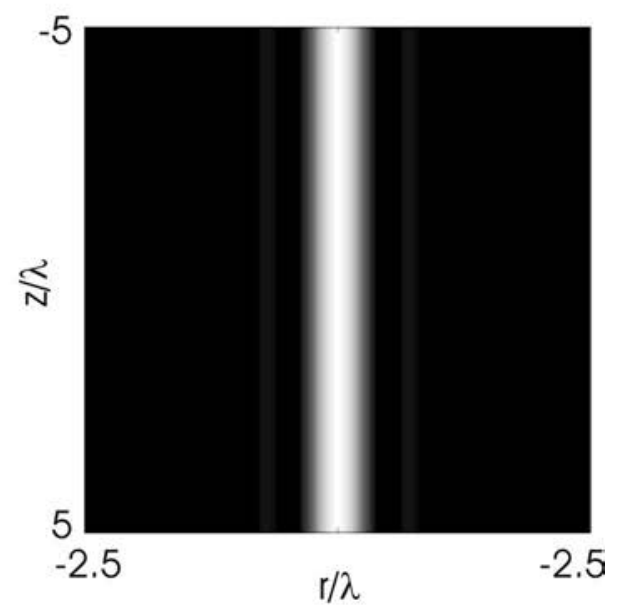

(d)

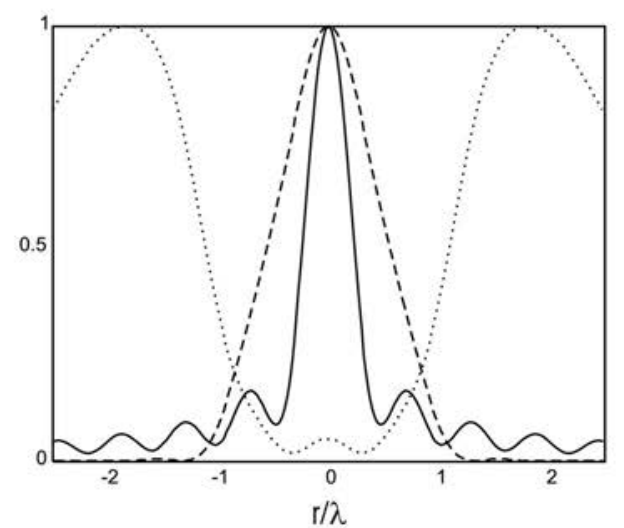

(e)

Fig. 2. Characterization of a radially polarized focused beam (a), (c) without angular frequency filtering and (b), (d) with focusing optimization, when $N A=\sin (\pi / 3)=0.87$. (a), (b) Intensity in the $(x, y)$ transverse plane; (c), (d) intensity in the $(x, z)$ longitudinal plane. (e) Comparison between the normalized profiles of (c) at $z=0$ (dashed curve) and $z=5 \lambda$ (dotted curve) and the normalized profile of (d) whatever value $z$.

sion of the probe-to-sample distance to the maximum without any loss of subwavelength confinement. Then the probe-to-sample coupling, which pollutes near-field image acquisition, can be avoided. The virtual tip establishes a link between confocal microscopy and scanning near-field optical microscopy (SNOM). Various fields of applications such as data storage and biology could benefit from such distance relaxations between probe and sample with highly confined fields. We see from Figs. 2(e) and 3(e) that the lateral fringes of the Bessel beams are stronger than those of usual focused beams. Unfortunately, this is the price to pay to obtain the smallest focal spots. 


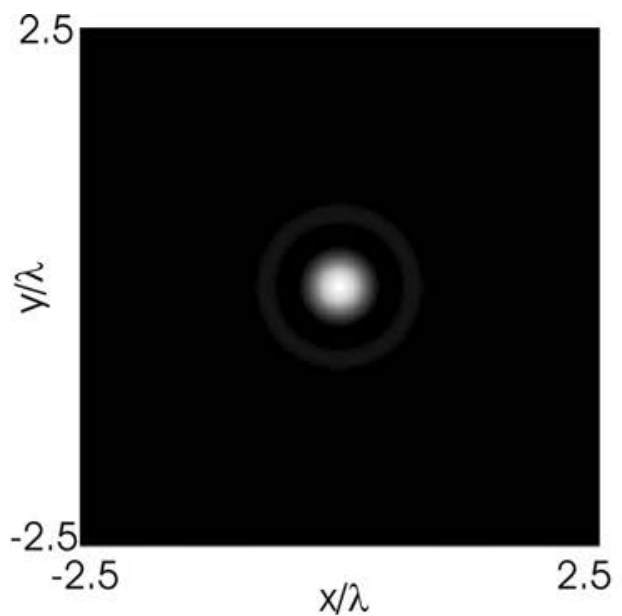

(a)

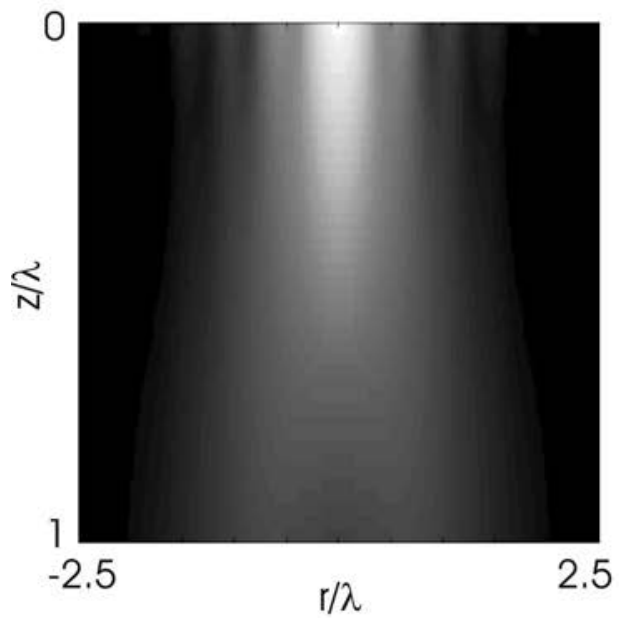

(c)

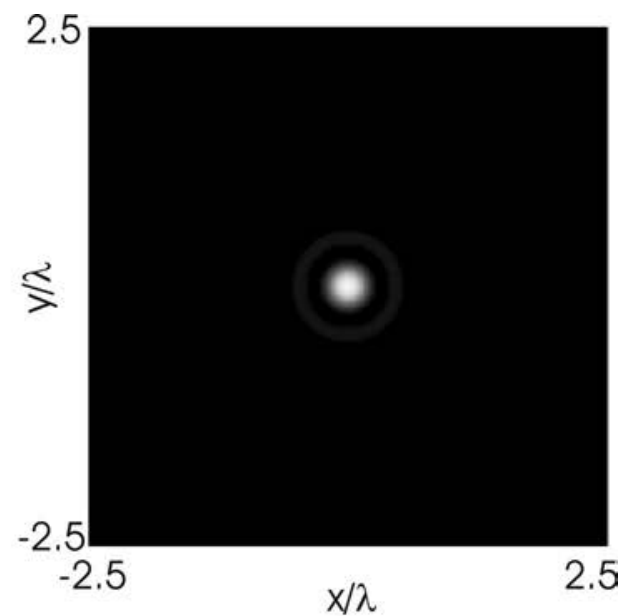

(b)

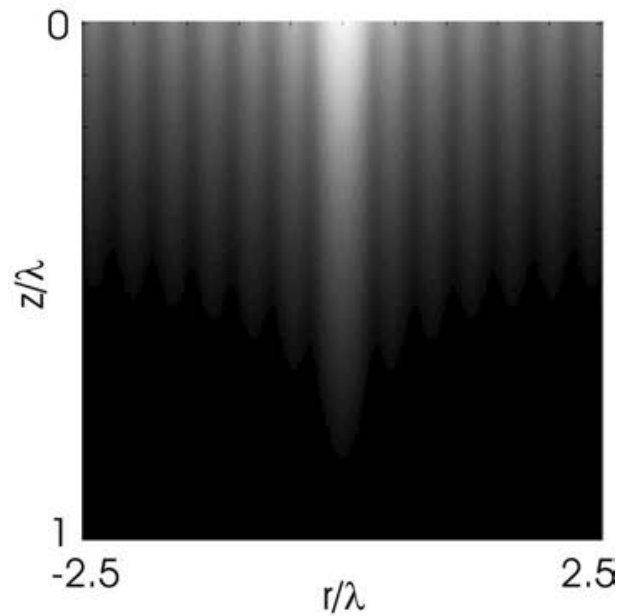

(d)

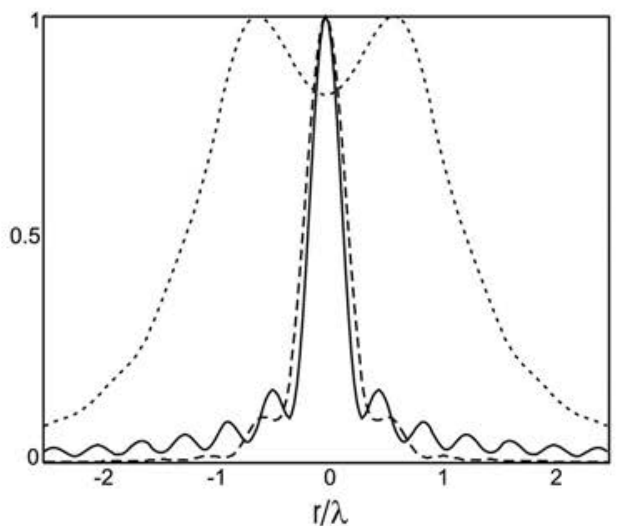

(e)

Fig. 3. Characterization of a radially polarized focused beam (a), (c) without angular frequency filtering and (b), (d) with focusing optimization, when $N A=1.5 \sin (\pi / 3)=1.73$. (a), (b) Intensity $(I)$ in the $(x, y)$ transverse plane; (c), (d) $I^{0.2}$ in the $(x, z)$ longitudinal plane. (e) Comparison between the normalized profiles of (c) at $z=0$ (dashed curve) and $z=\lambda$ (dotted curve) and the normalized profile of (d) whatever value $z$.

\section{Conical Optics: the Solution to Optimize the Focusing Process}

Spherical optics has adopted a masking process to generate Bessel beams. ${ }^{28,33-35}$ According to Fourier transform representation of microscopical imaging ${ }^{27}$ and reciprocity in optics, it is well known that the angular spectrum of a focused field objective is defined by the incident field distribution in the Fourier plane of the system. For microscope objectives, the Fourier plane of interest corresponds to the exit pupil 
plane. Using a ring-shape amplitude transmittance as a pupil plane filter induces the high-pass filtering process exposed in Section 3. Let us note that annular slits coupled to spherical optics have been previously studied in confocal microscopy in order to improve the resolution capability of the microscopes. ${ }^{36-39}$

However, Eq. (14) shows that the generation of Bessel beams in such a configuration requires the insertion of an infinitely thin annular slit (a circle) at the exit pupil plane of the objective. In that case, focusing optimization is obtained with a null efficiency. The realistic case in which the slit thickness is wider leads to the generation of Bessel-like beams ${ }^{33,34}$ with still very low efficiencies. Moreover, the phase relation between the Bessel beams, which constitute, by coherent superposition, Bessel-like beams, is such that a gain of confinement in the focusing process (annular slit shrinkage) is systematically counterbalanced by a dramatic loss of efficiency, and, conversely, a gain of efficiency (annular slit enlargement) is accompanied by a loss of confinement capability. A compromise between efficiency and optical confinement has to be found, depending on application, which involves some tricks in the search for highly confined light spots and superresolution. Therefore in the search for focusing optimization, spherical optics undergoes a fundamental limitation that makes it useless in practical application.

The way to overcome this problem is to generate Bessel beams with pure refractive systems. Cone lenses or axicons ${ }^{9}$ are then valuable solutions. They allow the accurate generation of highly confined Bessel beams from an incoming collimated beam, avoiding efficiency problems..$^{40,41}$ Here the width of the ring-shape angular spectrum of the Bessel beam is defined only by the divergence of the incident beam, no longer by the width of an annular pupil. By using collimated laser beams exhibiting a beam waist of several millimeters, the difference between the ideal Bessel beam of high NA and the Bessel beam generated with conical optics can be neglected (in terms of beam shape). Therefore Figs. 2 and 3 can be seen as the result of the simulation of the light fields generated by spherical and conical optics. It turns out that conical optics realizes with an efficiency close to unity what filtered spherical optics makes with a null efficiency. The combination of annular pupils and spherical lenses can be seen as a low-efficiency approximation of cone lenses. Therefore conical optics seems to be better adapted than spherical optics to the current and future optical needs and challenges associated with the general trend of system miniaturization.

The concept of high-NA conical objectives can be carried out through the proper combination of axicons in order to generate the smallest focus spots through focusing optimization. Let us note that the first realization of a solid immersion conical objective has recently been proposed for virtual tip generation. ${ }^{42}$ Conical interfaces have another advantage for focusing as they are less sensitive to abberations than spherical lenses. ${ }^{43}$ For example, axicons are not sub- ject to coma abberation. Let us note that conical optics has also been associated with spherical optics in the elaboration of a scanning microscope configuration. ${ }^{44}$ The cone lens has been used as a refractive system replacing the annular aperture. Such a microscope device can reach a high-efficiency focusing optimization. However, it gives much smaller focal depth than that provided by conical objectives.

\section{Conclusion}

Vectorial focused beams have been analyzed in detail to find the procedure that allows one to minimize the focus spot size, whatever the NA. It has been found, first, that the smallest light confinements (focusing optimization) and longest depth of focus produced by focusing are both carried by Bessel beams, and, second, that conventional microscope objectives (spherical optics) find their limit in Bessel beam generation. Although the well-known combination of objectives and annular masks yields to the achievement of the smallest focus spot, such systems are limited to very low efficiencies. Because the problem of efficiency for Bessel beam generation vanishes with cone lenses, spherical lenses appear to be less adapted than cone lenses to reach focusing optimization (and, by extension, parabolic mirrors are less adapted than conical mirrors). In many applications requiring highly confined fields, long depth of field, and/or high focus intensities, the conventional notion of microscope objectives should give way to the concept of conical objectives (the specific combination of axicons). Therefore one of the future challenges in optics manufacturing could be the development and improvement of fabrication and the optical treatment of conical interfaces. The first realization of a solid immersion conical objective has recently been proposed, and deeper investigations have to be realized. With such a system the generated light confinement acts as a nondiverging subwavelength stylus of light that can be applied as a near-field virtual tip for SNOM applications. Then conical optics bridges the gap between confocal microscopy and SNOM.

\section{References}

1. M. Staniforth, J. Goldstein, D. E. Newbury, C. E. Lyman, P. Echlin, E. Lifshin, L. C. Sawyer, J. R. Michael, and D. C. Joy, Scanning Electron Microscopy and X-Ray Microanalysis (Springer, 2002).

2. T. Wilson, ed., Confocal Microscopy (Academic, 1990).

3. J. Powell, $\mathrm{CO}_{2}$ Laser Cutting (Springer, 1998).

4. M. Will, S. Nolte, B. N. Chichkov, and A. Tünnermann, "Optical properties of waveguides fabricated in fused silica by femtosecond laser pulses," Appl. Opt. 21, 4360-4364 (2002).

5. Y. Kondo, K. Nouchi, T. Mitsuyu, M. Watanabe, P. G. Kazansky, and K. Hirao, "Fabrication of long-period fiber gratings by focused irradiation of infrared femtosecond laser pulses," Opt. Lett. 24, 646-648 (1999).

6. H.-B. Sun, Y. Xu, S. Juodkazis, K. Sun, M. Watanabe, S. Matsuo, H. Misawa, and J. Nishii, "Arbitrary-lattice photonic crystals created by multiphoton microfabrication," Opt. Lett. 26, 325-327 (2001).

7. L. P. Ghislain and V. B. Elings, "Near-field photolithography 
with a solid immersion lens," Appl. Phys. Lett. 74, 501-503 (1999).

8. T. D. Milster, "Horizons for optical data storage," Opt. Photon. News 16(3), 28-33 (2005).

9. J. H. McLeod, "Axicons and their uses," J. Opt. Soc. Am. 50, 166-169 (1960).

10. S. M. Mansfield and G. S. Kino, "Solid immersion microscope," Appl. Phys. Lett. 57, 2615-2616 (1990).

11. M. Born and E. Wolf, Principles of Optics, 6th ed. (Pergamon Press, 1980, reprinted Cambridge U. Press, 1997).

12. B. Richards and E. Wolf, "Electromagnetic diffraction in optical systems II. Structure of the image field in an aplanetic system," Proc. R. Soc. London Ser. A 253, 358-379 (1959).

13. J. J. Stammes, Waves in Focal Regions (Adam Hilger, 1986).

14. H. Weyl, "Ausbreitung elektromagnetischer Wellen über einem ebenen Leiter," Ann. Phys. (Leipzig) 60, 481-500 (1919).

15. Z. Bouchal and M. Olivik, "Non-diffractive vector Bessel beams," J. Mod. Opt. 42, 1555-1566 (1995).

16. P. Vahimaa, V. Kettunen, M. Kuittinen, J. Turunen, and A. T. Friberg, "Electromagnetic analysis of nonparaxial Bessel beams generated by diffractive axicon,” J. Opt. Soc. Am. A 14, 1817-1824 (1997).

17. T. Grosjean and D. Courjon, "Immaterial tip concept by light confinement," J. Microsc. (Oxford) 202, 273-278 (2001).

18. J. Durnin, "Exact solutions for nondiffracting beams. i. The scalar theory," J. Opt. Soc. Am. A 4, 651-654 (1987).

19. M. Zamboni-Rached, A. M. Shaarawi, and E. Recami, "Focused x-shaped pulses," J. Opt. Soc. Am. A 21, 1564-1574 (2004).

20. G. Toraldo di Francia, "Supergain antennas and optical resolving power," Nuovo Cimento, Suppl. 9, 426-435 (1952).

21. Z. S. Hegedus, "Annular pupil arrays. Application to confocal scanning," Opt. Acta 32, 815-826 (1985).

22. Z. S. Hegedus, "Superresolving filters in confocally scanned imaging systems," J. Opt. Soc. Am. A 3, 1892-1896 (1986).

23. M. Martinez-Corral, P. Andres, C. J. Zapata-Rodriguez, and M. Kowalczyk, "Three-dimensional superresolution by annular binary filters," Opt. Commun. 165, 267-278 (1999).

24. M. A. A. Neil, R. Juskaitis, T. Wilson, and Z. J. Laczik, "Optimized pupil-plane filters for confocal microscope point-spread function engineering," Opt. Lett. 25, 245-247 (2000).

25. I. Leiserson, S. G. Lipson, and V. Sarafis, "Superresolution in far-field imaging," Opt. Lett. 25, 209-211 (2000).

26. C.-C. Sun and C.-K. Liu, "Ultrasmall focusing spot with a long depth of focus based on polarization and phase modulation," Opt. Lett. 28, 99-101 (2003).

27. M. Pluta, Advanced Light Microscopy Vol. I: Principles and Basic Properties (Elsevier, 1988).
28. J. Durnin, J. J. Miceli, and J. H. Eberly, "Diffraction-free beams," Phys. Rev. Lett. 58, 1499-1501 (1987).

29. F. Gori, G. Guattari, and C. Padovani, "Bessel-Gauss beams," Opt. Commun. 64, 491-495 (1987).

30. P. L. Overfelt and C. S. Kenney, "Comparison of the propagation characteristics of Bessel, Bessel-Gauss, and Gaussian beams diffracted by a circular aperture," J. Opt. Soc. Am. A 8, 732-745 (1991).

31. Z. Jiang, Q. Lu, and Z. Liu, "Propagation of apertured Bessel beams," Appl. Opt. 34, 7183-7185 (1996).

32. S. Quabis, R. Dorn, M. Eberler, O. Glöckl, and G. Leuchs, "Focusing light to a tighter spot," Opt. Commun. 179, 1-7 (2000).

33. T. Grosjean and D. Courjon, "Polarization filtering induced by imaging systems: effect on image structure," Phys. Rev. E 67, 46611 (2003).

34. C. J. R. Sheppard and A. Choudhury, "Annular pupils, radial polarization, and superresolution," Appl. Opt. 43, 4322-4327 (2004).

35. A. Burvall, K. Kolacz, Z. Jaroszewicz, and A. T. Friberg, "Simple lens axicon," Appl. Opt. 43, 4838-4844 (2004).

36. C. J. R. Sheppard and A. Choudhury, "Image formation in the scanning microscope," Opt. Acta 24, 1051-1073 (1977).

37. C. J. R. Sheppard, "The use of lenses with annular aperture in scanning optical microscopy," Optik (Stuttgart) 48, 329-334 (1977).

38. M. Gu, T. Tannous, and C. J. R. Sheppard, "Effect of an annular pupil on confocal imaging through highly scattering media," Opt. Lett. 21, 312-314 (1996).

39. S. Lindek, C. Cremer, and E. H. Stelzer, "Confocal theta fluorescence microscopy with annular apertures,” Appl. Opt. 35, 126-130 (1996).

40. R. M. Herman and T. A. Wiggins, "Apodization of diffractionless beams,” Appl. Opt. 31, 5913-5915 (1992).

41. G. Scott and N. McArdle, "Efficient generation of nearly diffraction-free beams using an axicon," Opt. Eng. 31, 26402643 (1992).

42. T. Grosjean, D. Courjon, and D. Van Labeke, "Bessel beams as virtual tips for near-field optics," J. Microsc. (Oxford) 210, 319-323 (2003).

43. T. Tanaka and S. Yamamoto, "Comparison of abberation between axicon and lens," Opt. Commun. 184, 113-118 (2000).

44. R. Arimoto, C. Saloma, T. Tanaka, and S. Kawata, "Imaging properties of axicon in a scanning optical system," Appl. Opt. 31, 6653-6657 (1992). 